\title{
Characterization of gait variability in multiple system atrophy and Parkinson's disease
}

\author{
Victoria Sidoroff $^{1} \cdot$ Cecilia Raccagni ${ }^{1,6}$ (1) Christine Kaindlstorfer $^{1} \cdot$ Sabine Eschlboeck ${ }^{1}$. Alessandra Fanciulli ${ }^{1}$. \\ Roberta Granata $^{1}$ - Björn Eskofier ${ }^{2} \cdot$ Klaus Seppi $^{1}$ - Werner Poewe ${ }^{1}$. Johann Willeit ${ }^{1}$. Stefan Kiechl ${ }^{1}$. \\ Philipp Mahlknecht ${ }^{1}$. Heike Stockner ${ }^{1}$ - Kathrin Marini ${ }^{1}$. Oliver Schorr ${ }^{1}$. Gregorio Rungger ${ }^{3}$. Jochen Klucken ${ }^{4,5}$. \\ Gregor Wenning ${ }^{1} \cdot$ Heiko Gaßner ${ }^{4}$
}

Received: 25 June 2020 / Revised: 7 October 2020 / Accepted: 4 December 2020 / Published online: 31 December 2020

(c) The Author(s) 2020

\begin{abstract}
Background Gait impairment is a pivotal feature of parkinsonian syndromes and increased gait variability is associated with postural instability and a higher risk of falls.

Objectives We compared gait variability at different walking velocities between and within groups of patients with Parkinsonvariant multiple system atrophy, idiopathic Parkinson's disease, and a control group of older adults.

Methods Gait metrics were recorded in 11 multiple system atrophy, 12 Parkinson's disease patients, and 18 controls using sensor-based gait analysis. Gait variability was analyzed for stride, swing and stance time, stride length and gait velocity. Values were compared between and within the groups at self-paced comfortable, fast and slow walking speed.

Results Multiple system atrophy patients displayed higher gait variability except for stride time at all velocities compared with controls, while Parkinson's patients did not. Compared with Parkinson's disease, multiple system atrophy patients displayed higher variability of swing time, stride length and gait velocity at comfortable speed and at slow speed for swing and stance time, stride length and gait velocity (all $P<0.05$ ). Stride time variability was significantly higher in slow compared to comfortable walking in patients with multiple system atrophy $(P=0.014)$. Variability parameters significantly correlated with the postural instability/gait difficulty subscore in both disease groups. Conversely, significant correlations between variability parameters and MDS-UPDRS III score was observed only for multiple system atrophy patients.

Conclusion This analysis suggests that gait variability parameters reflect the major axial impairment and postural instability displayed by multiple system atrophy patients compared with Parkinson's disease patients and controls.
\end{abstract}

Keywords Parkinson's disease $\cdot$ Multiple system atrophy $\cdot$ Gait variability $\cdot$ Gait analysis $\cdot$ Wearable sensors

\section{Introduction}

Gait impairment, reduced mobility and falls are axial motor complications of parkinsonian disorders, more pronounced and less responsive to treatment in patients

Victoria Sidoroff and Cecilia Raccagni contributed equally to the manuscript.

Supplementary Information The online version contains supplementary material available at https://doi.org/10.1007/s0041 5-020-10355-y.

Cecilia Raccagni

cecilia.raccagni@sabes.it

Extended author information available on the last page of the article with Parkinson variant multiple system atrophy (MSA-P) than in patients with idiopathic Parkinson's disease (PD) $[1,2]$. Clinimetric rating scales such as the Movement Disorder Society revision of the Unified Parkinson's Disease Rating Scale (MDS-UPDRS) or the Unified Multiple System Atrophy Rating Scale (UMSARS) show excellent construct validity in quantifying PD-related signs, nonetheless they provide very little information about gait impairment. For this reason, a prominent research effort over the last decades was centred on the development and validation of motion analysis technology. Importantly, senso-based gait analysis by means of inertial measurement units (IMUs) has proven to be a usable supplementary device with high sensitivity and specificity for assessing gait performance [3]. Patient-centred, 
wearable-derived parameters have the potential to trace prodromal axial signs, support differential diagnosis and monitor the responsiveness of treatment during interventional trials $[1,4]$.

To date, several studies have investigated the feasibility of IMUs in PD patients, and their clinical applicability has become evident [5]. The locomotor impairment of PD patients is reflected by alterations of gait velocity, cadence, stride time and stride length, showing progression throughout the disease course [6]. Another important domain of gait functioning is represented by its variability.

Gait variability $(\mathrm{GV})$ is defined as the percent range of variance describing the regularity and consistency of a step cycle that is directly linked to dynamic postural control [7, 8]. Key measurements include variability of stride, swing and stance time, as well as stride length and gait velocity. A high GV reflects altered walking in terms of magnitude and dynamics mainly observed in movement disorders including PD [7] and Huntington's disease [8]. Several studies demonstrated that a high GV results in walking instability and a higher risk of falling in the general elderly population [9] and in patients with PD [8]. An increased stance time and swing time variability-together with an abnormal average gait velocity-predict a higher risk of falls in PD patients regardless of medical treatment [10].

While several studies mainly focused on PD patients, only a few data have been reported for atypical parkinsonism including MSA-P. A quantitative analysis of gait parameters revealed a significant reduction of average gait velocity and stride length in patients with atypical parkinsonism compared to PD patients [11] and further observations also demonstrated increased GV [1]. Previous research described a disease-characteristic balance impairment in the medio-lateral axis displayed by patients with atypical parkinsonism [12], which is unveiled by an abnormal tandem gait test and by the information that patients stop riding a bicycle $[13,14]$. However, detailed information about $\mathrm{GV}$-as well as the impact of walking velocity on GV-in MSA-P patients is lacking. Although inertial sensors are not able to measure gait width and its variability yet, the available gait parameters might give us relevant insights into gait patterns of these patients.

In this study, we tested the hypothesis that MSA-P patients show a higher instability of walking reflected by an increased variability of spatio-temporal gait parameters at different gait velocities compared to PD patients and parkinsonism-free older adults of similar age. Secondly, we investigated whether walking at different velocities affects the stability of gait, evaluating gait parameters at self-paced comfortable, fast and slow walking speed between and within the groups.

\section{Patients and methods}

This study is a post-hoc analysis of 23 patients (MSA-P $n=11$, PD $n=12$ ) enrolled in the outpatient clinic of the Department of Neurology at the Medical University Hospital of Innsbruck between June 2017 and March 2019 [4]. The study was approved by local ethics committees (IRB-approval-No 0365, 344/4.25 378/5.3, 20.10.2017) and all study participants gave written informed consent prior to participation according to the Declaration of Helsinki. Disease groups were matched by gender and Hoehn and Yahr stage, the overall inclusion and exclusion criteria are described elsewhere [4]. Briefly, the diagnosis of PD was confirmed by using the MDS criteria [15] and probable MSA-P by Gilman criteria [16]. A general and neurological examination was performed and the motor/ non-motor impairment on medication was assessed using the MDS-UPDRS for PD and MSA-P patients [17], and the UMSARS for MSA-P patients [18]. To focus on gait specific items, postural instability and gait difficulty (PIGD) subscores, were calculated as sum of the items "arising from chair", "gait", "postural stability" and "posture" from MDS-UPDRS III [1, 19]. Specific balance testing including the Berg Balance Scale and the Timed Up and Go Test were performed and are reported elsewhere [4]. Patients were regularly followed over at least 24 months to reassess their clinical diagnosis, and one case was reclassified as PD during clinical follow-up. The control group consisted of older adults assessed within an ongoing extension of the population-based Bruneck Study [20] performed at the Bruneck Hospital, Bolzano, Italy. Controls were clinically assessed according to a protocol used in previous Bruneck Study assessments including the MDSUPDRS [21] and underwent sensor-based gait analysis as detailed below. Only controls free of parkinsonism and without clinically apparent gait impairment and postural instability - as measured by the corresponding items of MDS-UPDRS III-that were in the same age range as PD patients (65-80 years) were included in the present analysis $(n=18)$.

The gait trials for every participant consisted of three consecutive tasks performed uninterrupted on an overground $20 \mathrm{~m}$ distance $(2 \times 10 \mathrm{~m}$ walk), respectively at (a) self-paced comfortable, (b) fast, and (c) slow walking velocity. The walking pace was performed without using external cues to reflect the patients' natural gait modulation and was done in the same order in every patient.

A detailed description of the used technical device is provided elsewhere [4, 22]. Briefly, instrumented gait analysis was performed using in-sole wearable sensors (Shimmer Research Ltd., Dublin, Ireland). Collected data was extracted by a pattern recognition algorithm for 
calculating clinically relevant spatio-temporal gait parameters including stride, swing and stance time as well as stride length and gait velocity (reported as the average of strides from left and right foot). We conducted a quality check of the collected data by manually reviewing the parameters for each patient and eliminating the potential technically-induced or not matching outliers. Each outlier deviating more than two standard deviations from the mean values was evaluated individually. From overall 3416 strides (on average 26.5 strides per patient per gait test), 9 strides (maximum two strides per patient per gait test) were excluded due to inconsistency or implausible values $(0.44 \%)$. The coefficient of variation (CV) representing the variability of gait was calculated for the parameters stride time, percent swing and stance time, stride length and gait velocity. Mean values for stride length and gait velocity were calculated as well.

Statistical analysis was performed using IBM SPSS Statistics 24 (IBM Corp., Armonk NY). Due to non-normally distributed parameters as shown by the Kolmogorov-Smirnov test, descriptive statistics were compared using Kruskal-Wallis-Test for total $P$-values and Mann Whitney $\mathrm{U}$ test to compare between groups. The significance level was set at two-sided and adapted by Bonferroni correction for multiple testing. $P$-values $<0.007$ were considered as statistically significant. For each gait parameter, a one-way ANOVA and Bonferroni post-hoc test were performed for group comparison. Spearman correlation analysis was conducted between gait parameters and MDS-UPDRS III as well as PIGD subscores. For within-group analysis, a oneway ANOVA for repeated measures followed by Bonferroni post-hoc test was performed.

\section{Results}

\section{Patient and participant's characteristics}

The demographic data and clinical characteristics of the participants are presented in Table 1.

All participants were able to perform the gait tasks properly without falling. The sensor-based gait analysis system automatically recognized strides for each cohort in each walking condition and stride count similarly changed with gait speed modulation within the cohorts: comfortable speed-MSA 32, PD 27, CG 25 strides, fast speed-MSA 30, PD 22, CG 19 strides, and slow speed-MSA 39, PD 27, CG 26 strides.

\section{Gait parameters}

\section{Between-group comparison}

A detailed description of the $\mathrm{CV}$ parameters for the different groups is reported in Table 2. Figure 1 illustrates the comparison of the spatio-temporal parameters between the groups.

\section{Comfortable speed}

The mean gait velocity was significantly reduced in MSA-P patients compared to controls. No differences were found between the MSA-P and PD group. Stride length was significantly larger in the control group compared to MSA-P and PD but there was no difference between the disease groups.

Table 1 Demographics and clinical characteristics in the disease groups

\begin{tabular}{|c|c|c|c|c|c|c|c|}
\hline & $\begin{array}{l}\text { MSA-P } \\
(n=11)\end{array}$ & $\begin{array}{l}\mathrm{PD} \\
(n=12)\end{array}$ & $\begin{array}{l}\text { CG } \\
(n=18)\end{array}$ & $P$ value & $\begin{array}{l}P \text { value } \\
\text { MSA-P vs. PD }\end{array}$ & $\begin{array}{l}P \text { value } \\
\text { MSA-P vs. CG }\end{array}$ & $\begin{array}{l}P \text { value } \\
\text { PD vs. CG }\end{array}$ \\
\hline Gender (m:f) & $5: 6$ & $6: 6$ & $13: 5$ & 0.292 & 0.880 & 0.238 & 0.325 \\
\hline Age & $56.0(55 ; 59)$ & $74.5(63.75 ; 77)$ & $75.5(74.75 ; 78)$ & $<0.001$ & $<0.001$ & $<0.001$ & 0.134 \\
\hline Falls last 12 months $(\mathrm{N})$ & $3(1 ; 10)$ & $1(0 ; 1)$ & 0 & $<0.001$ & 0.009 & $<0.001$ & 0.013 \\
\hline Disease duration (years) & $4(3 ; 6)$ & $7.5(3.5 ; 11.5)$ & - & $<0.001$ & $\mathbf{0 . 0 3 7}$ & - & - \\
\hline MDS-UPDRS II & $22(14.5 ; 28.25)$ & $7(3.25 ; 9)$ & $1(0 ; 2)$ & $<0.001$ & $<0.001$ & $<0.001$ & $<0.001$ \\
\hline MDS-UPDRS III & $36(20 ; 62)$ & $21.5(16.25 ; 34)$ & $5(1.75 ; 7.25)$ & $<0.001$ & 0.059 & $<0.001$ & $<0.001$ \\
\hline MDS-UPDRS total score & $75(47.25 ; 83.75)$ & $38.5(26.25 ; 49.75)$ & $11(7.5 ; 15.25)$ & $<0.001$ & 0.002 & $<0.001$ & $<0.001$ \\
\hline PIGD subscore & $4(1.5 ; 6)$ & $6.5(3 ; 10.25)$ & 0 & $<0.001$ & 0.069 & $<0.001$ & $<0.001$ \\
\hline UMSARS II & $22(14 ; 33)$ & - & - & - & - & - & - \\
\hline Hoehn and Yahr stage & 3 & $2.5(2 ; 3)$ & - & $<0.001$ & 0.190 & - & - \\
\hline LED (mg/day) & $0(0 ; 400)$ & $600(325 ; 858.75)$ & - & $<0.001$ & 0.044 & - & - \\
\hline
\end{tabular}

Kruskal-Wallis-Test, significance level $P<0.05$; All values are specified as median and interquartile range (25th and 75th percentiles). Bold numbers indicate significance

MSA-P Parkinsonian variant of Multiple system atrophy, $P D$ Parkinson's disease, $C G$ control group, $M D S$ - UPDRS MDS-sponsored revision of the Unified Parkinson's Disease Rating Scale, PIGD postural instability and gait difficulty, UMSARS Unified Multiple System Atrophy Rating Scale, LED Levodopa equivalent dose 
Table 2 Mean gait parameters and gait variability parameters at comfortable, fast and slow walking velocity

\begin{tabular}{|c|c|c|c|c|c|c|c|}
\hline & \multicolumn{3}{|c|}{ Mean values } & \multicolumn{4}{|l|}{$P$ values } \\
\hline & MSA-P & PD & $\mathrm{CG}$ & Total & MSA-P vs. PD & MSA-P vs. CG & PD vs. CG \\
\hline \multicolumn{8}{|l|}{ Comfortable speed } \\
\hline \multicolumn{8}{|l|}{ Variability } \\
\hline Stride time CV (\%) & $4.922 *$ & 3.440 & 3.819 & 0.118 & 0.146 & 0.319 & 1.0 \\
\hline Swing time $\mathrm{CV}(\%)$ & 7.546 & 4.520 & 3.929 & 0.005 & 0.040 & 0.005 & 1.0 \\
\hline Stance time CV (\%) & 3.509 & 2.430 & 2.219 & 0.016 & 0.087 & 0.016 & 1.0 \\
\hline Stride length CV (\%) & 10.588 & 6.101 & 5.089 & $<0.001$ & $<0.001$ & $<0.001$ & 0.827 \\
\hline Gait velocity CV (\%) & 11.668 & 6.896 & 6.207 & $<0.001$ & 0.002 & $<0.001$ & 1.0 \\
\hline \multicolumn{8}{|l|}{ Mean } \\
\hline Stride length $(\mathrm{cm})$ & 92.61 & 112.09 & 132.94 & $<0.001$ & 0.069 & $<0.001$ & 0.021 \\
\hline Gait velocity $(\mathrm{m} / \mathrm{s})$ & 0.792 & 0.999 & 1.199 & $<0.001$ & 0.109 & $<0.001$ & 0.074 \\
\hline \multicolumn{8}{|l|}{ Fast speed } \\
\hline \multicolumn{8}{|l|}{ Variability } \\
\hline Stride time CV (\%) & 4.842 & 4.103 & 4.146 & 0.455 & 0.825 & 0.786 & 1.0 \\
\hline Swing time $\mathrm{CV}(\%)$ & 7.541 & 5.291 & 3.813 & 0.001 & 0.106 & 0.001 & 0.350 \\
\hline Stance time CV (\%) & 4.086 & 3.265 & 2.350 & 0.005 & 0.427 & 0.004 & 0.207 \\
\hline Stride length CV (\%) & 9.911 & 7.004 & 5.569 & 0.001 & 0.051 & 0.001 & 0.527 \\
\hline Gait velocity CV (\%) & 11.285 & 8.333 & 6.631 & 0.003 & 0.118 & 0.002 & 0.527 \\
\hline \multicolumn{8}{|l|}{ Mean } \\
\hline Stride length $(\mathrm{cm})$ & 104.02 & 130.36 & 150.00 & $<0.001$ & 0.017 & $<0.001$ & 0.057 \\
\hline Gait velocity $(\mathrm{m} / \mathrm{s})$ & 1.007 & 1.339 & 1.521 & $<0.001$ & 0.013 & $<0.001$ & 0.221 \\
\hline \multicolumn{8}{|l|}{ Slow speed } \\
\hline \multicolumn{8}{|l|}{ Variability } \\
\hline Stride time CV (\%) & $6.005^{*}$ & 4.451 & 4.281 & 0.102 & 0.271 & 0.127 & 1.0 \\
\hline Swing time CV (\%) & 9.067 & 5.059 & 4.108 & $<0.001$ & $<0.001$ & $<0.001$ & 0.803 \\
\hline Stance time CV (\%) & 4.013 & 2.475 & 2.209 & $<0.001$ & 0.001 & $<0.001$ & 1.0 \\
\hline Stride length CV (\%) & 12.187 & 7.145 & 5.562 & $<0.001$ & 0.003 & $<0.001$ & 0.641 \\
\hline Gait velocity CV (\%) & 13.352 & 8.035 & 7.356 & 0.001 & 0.007 & 0.001 & 1.0 \\
\hline \multicolumn{8}{|l|}{ Mean } \\
\hline Stride length $(\mathrm{cm})$ & 80.92 & 102.94 & 121.72 & $<0.001$ & 0.040 & $<0.001$ & 0.053 \\
\hline Gait velocity (m/s) & 0.625 & 0.814 & 0.950 & 0.001 & 0.105 & 0.001 & 0.255 \\
\hline
\end{tabular}

ANOVA followed by Bonferroni post-hoc test. Values in bold are marked as significant (significance level was adapted by Bonferroni correction for multiple testing, $P<0.007$ )

$C V$ coefficient of variation, $M S A-P$ Parkinsonian variant of Multiple system atrophy, $P D$ Parkinson's disease, $C G$ control group

*Asterisks indicate within-group differences across gait speed conditions
Regarding the CV parameters, MSA-P patients showed a significantly higher variability of swing time, gait velocity and stride length $(P<0.001)$ compared to PD patients and all values except stride time showed statistical difference between MSA-P and controls (see Fig. 1).

\section{Fast speed}

Average gait velocity was reduced in MSA-P patients compared to PD and controls. Mean stride length was significantly higher in PDs and controls compared to MSA-P patients. As in comfortable speed, MSA-P patients showed higher $\mathrm{CV}$ of all parameters except stride time compared to the control group, respectively. There were no significant differences of CV parameters between PDs and MSA-Ps as well as between PDs and controls (see Fig. 1).

\section{Slow speed}

Mean gait velocity was significantly reduced in MSA-P patients vs. controls, but not between the disease groups. 
Fig. 1 Group comparison of variability of stride length and gait velocity at different gait speeds

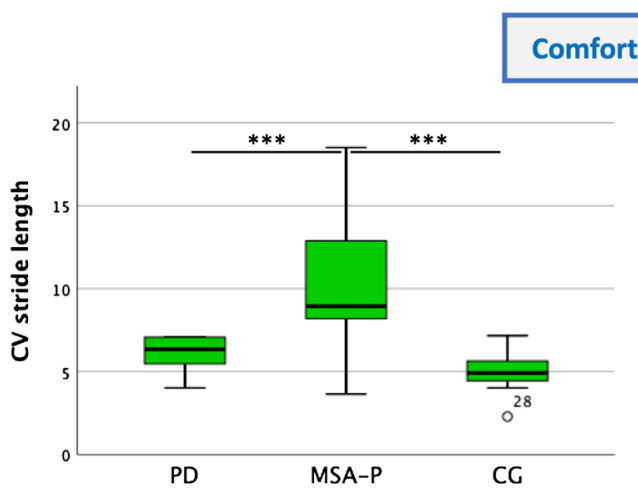

Comfortable velocity

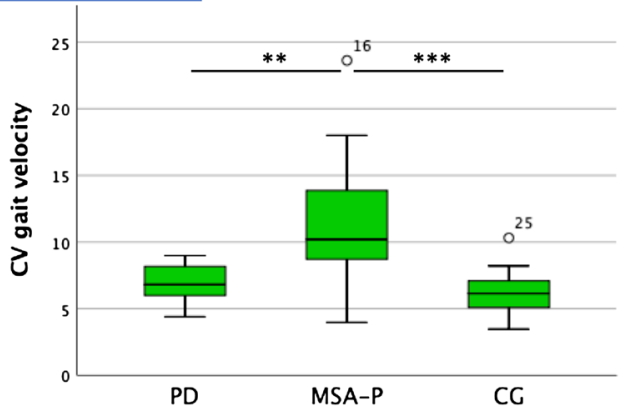

Fast velocity
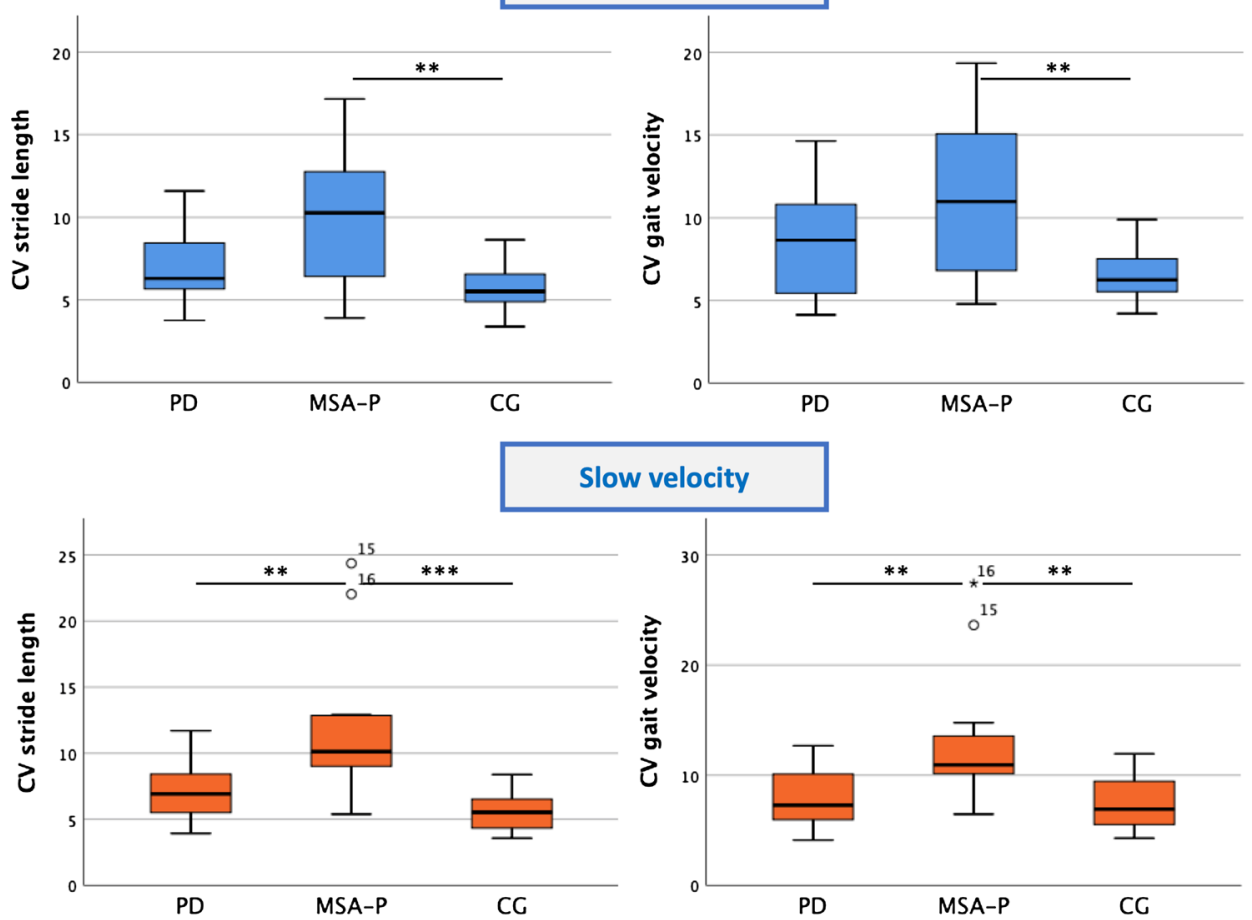

Mean stride length was significantly higher in the control group than in the disease groups and in PD compared to MSA-P. As illustrated in Fig. 1, MSA-P patients showed higher values in all CV parameters except stride time compared to PD and controls. Again, no significant differences were observed for GV parameters between PD and controls.

\section{Within-group analysis}

A detailed breakdown of the modulation of mean gait velocity as well as of mean stride length are reported in
Fig. 2. All study participants were able to significantly modulate their walking velocity according to the tasks (within-group differences $P<0.001$ in every group for every task). Large inter-individual differences for gait velocity adaptations were observed (mean ranges comfortable-slow: $0.167 \mathrm{~m} / \mathrm{s}$ in MSA-P, $0.185 \mathrm{~m} / \mathrm{s}$ in PD, $0.249 \mathrm{~m} / \mathrm{s}$ in controls; comfortable-fast: $0.214 \mathrm{~m} / \mathrm{s}$ in MSA-P, $0.341 \mathrm{~m} / \mathrm{s}$ in PD, $0.322 \mathrm{~m} / \mathrm{s}$ in controls).

While self-paced fast gait velocity in MSA-P showed mean values of $1.01 \mathrm{~m} / \mathrm{s}$, the same velocity was used by PDs for comfortable speed $(0.99 \mathrm{~m} / \mathrm{s})$ and by OAs even as slow speed $(0.95 \mathrm{~m} / \mathrm{s})$. 


\section{Mean gait velocity $(\mathrm{m} / \mathrm{s})$}

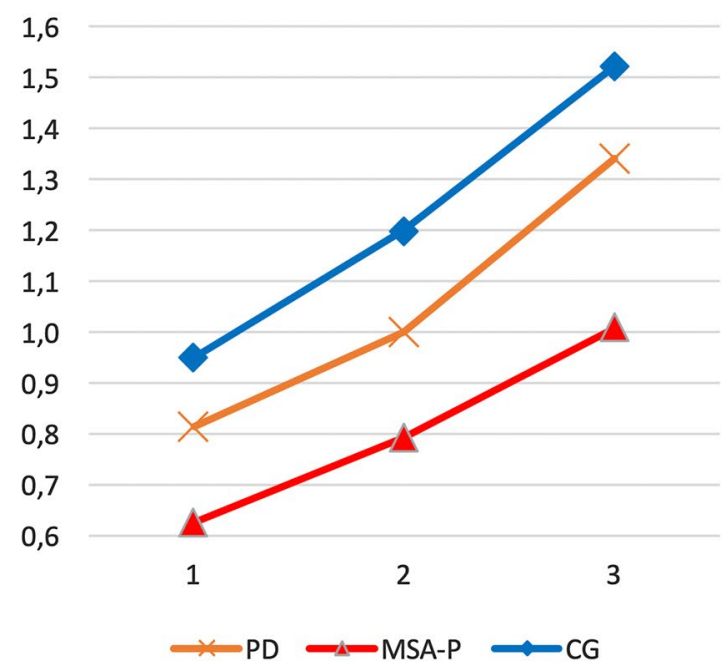

Fig. 2 Mean gait velocity and stride length of all groups

To analyze the relative speed modulation in each group, we calculated the ratio between the velocity transitions (comfortable-to-fast $=\frac{\text { fast }- \text { comfortable }}{\text { comfortale }} * 100$; comfortableto-slow $\left.=\frac{\text { comfortable }- \text { slow }}{\text { comfortable }} * 100\right)$ and observed higher ranges in PD patients compared to MSA-P patients and controls (see supplementary Fig. 1).

The highest variability of gait was displayed at slow speed in MSA patients (see Table 2), where we observed a numerical increase of all $\mathrm{CV}$ parameters, which reached statistical significance for stride time $\mathrm{CV}$ in comfortable to slow speed in MSA-P patients $(P=0.014$; effect power 0.688; see supplementary Fig. 2). Conversely, PD and controls did not show any significant differences of all CV parameters between the three gait trials.

\section{Correlation of CV with MDS-UPDRS III/PIGD subscore}

The correlation between gait metrics and the MDS-UPDRS III scores can be reviewed in detail in the supplementary Table 1. Correlation analysis with MDS-UPDRS III was significant for all mean and CV gait parameters at a comfortable speed in MSA-P patients, while in PD patients the scores only correlated with CV stride length. At other gait velocities, inverse correlations were observed in MSA-P patients but not in PD patients.

Correlations between gait metrics and PIGD subscores are listed in Table 3. In both MSA-P and PD patients PIGD subscores showed significant correlations with $\mathrm{CV}$ and mean gait parameters in comfortable and slow speed.

\section{Mean stride length $(\mathrm{cm})$}

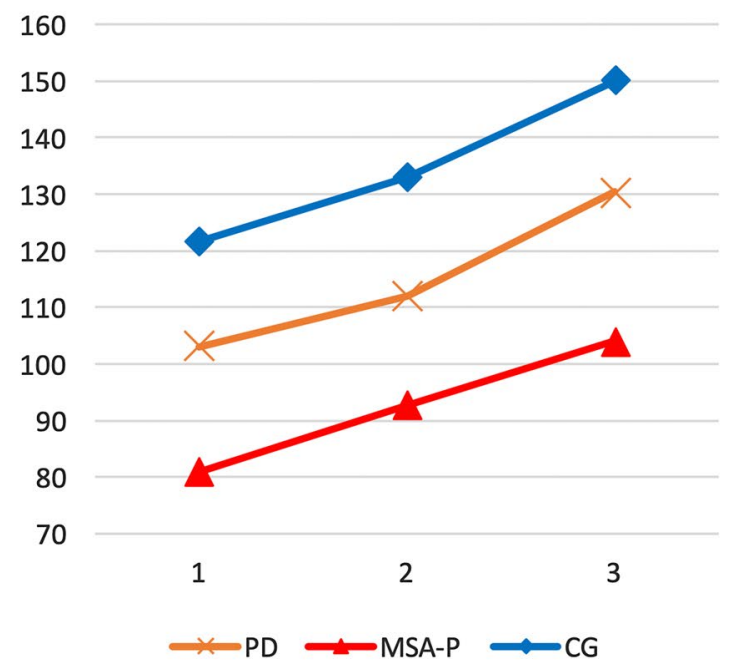

Table 3 Correlation analysis between PIGD scores and gait parameters

\begin{tabular}{|c|c|c|c|c|}
\hline & \multicolumn{2}{|c|}{ PIGD-MSA } & \multicolumn{2}{|l|}{ PIGD-PD } \\
\hline & Rho & $P$ value & Rho & $P$ value \\
\hline \multicolumn{5}{|l|}{ Comfortable speed } \\
\hline CV Stride time & 0.794 & 0.006 & 0.392 & 0.208 \\
\hline CV Swing time & 0.754 & 0.013 & 0.619 & 0.032 \\
\hline CV Stance time & 0.782 & 0.008 & 0.406 & 0.191 \\
\hline CV Stride length & 0.609 & 0.061 & 0.641 & 0.025 \\
\hline CV Gait velocity & 0.462 & 0.179 & 0.456 & 0.137 \\
\hline Stride length & -0.609 & 0.061 & -0.740 & 0.006 \\
\hline Gait velocity & -0.652 & 0.041 & -0.929 & $<0.001$ \\
\hline \multicolumn{5}{|l|}{ Fast speed } \\
\hline CV Stride time & 0.622 & 0.055 & 0.235 & 0.462 \\
\hline CV Swing time & 0.732 & 0.016 & 0.043 & 0.895 \\
\hline CV Stance time & 0.769 & 0.009 & 0.100 & 0.758 \\
\hline CV Stride length & 0.462 & 0.179 & 0.263 & 0.408 \\
\hline CV Gait velocity & 0.351 & 0.320 & 0.149 & 0.643 \\
\hline Stride length & -0.782 & 0.008 & -0.662 & 0.019 \\
\hline Gait velocity & -0.837 & 0.003 & -0.740 & 0.006 \\
\hline \multicolumn{5}{|l|}{ Slow speed } \\
\hline CV Stride time & 0.702 & 0.024 & 0.491 & 0.105 \\
\hline CV Swing time & 0.499 & 0.142 & 0.7444 & 0.006 \\
\hline CV Stance time & 0.665 & 0.036 & 0.673 & 0.017 \\
\hline CV Stride length & 0.523 & 0.121 & 0.705 & 0.010 \\
\hline CV Gait velocity & 0.628 & 0.052 & 0.587 & 0.045 \\
\hline Stride length & -0.652 & 0.041 & -0.562 & 0.057 \\
\hline Gait velocity & -0.726 & 0.017 & -0.760 & $<0.001$ \\
\hline
\end{tabular}

Spearman's correlation coefficient (rho) calculated for MSA-P and PD patients. Bold values are marked as significant 


\section{Discussion}

In this study, we tested the hypothesis that MSA-P patients display higher variability of gait at different gait velocities compared to PD patients and controls. Secondly, we investigated the within-group differences of gait metrics at three different self-paced walking velocities and correlated the clinical rating scales with the $\mathrm{CV}$ parameters.

One key finding of the present work is that MSA-P patients display higher GV than PD patients and controls and these differences are more distinct in slow speed, compared to comfortable or fast speed. Previous research indicated that patients with atypical parkinsonism including — but not limited to-MSA-P show significantly reduced mean gait velocity and stride length and even more pronounced differences in variability parameters compared to PD patients [1]. According to these findings, our study demonstrated that nearly all CV parameters measured in MSA-P patients were different from those obtained in PD and controls, revealing an overall greater instability of gait. The fact that mean gait velocity was not substantially different at a comfortable speed in MSA-P compared to PD while GV parameters were significantly larger in MSA-P, may indicate that GV parameters represent a more sensitive discrimination marker. Importantly, this study generates novel insights into parkinsonian gait at different walking velocities. Here, impaired balance and modulation of gait speed were demonstrated for slow and partially for comfortable but not for fast walking velocity in MSA-P compared to PD. The results of previous research exploring the influence of different walking speeds on gait variability are controversial. A number of studies in other cohorts suggested slow walking to be directly correlated with an increase in gait variability because it requires more stability in the medio-lateral axis [23]. Here, one might argue that this phenomenon may reflect the major impairment of balance affecting MSA-P patients [12]. As a consequence, tandem gait cannot be performed in the vast majority of MSA-P patients [13] and riding a bike results very difficult even in early stages [14]. However, data about the impact of fast walking are unclear. A study showed that fast walking does not increase gait variability in patients with PD, corroborating our results [24]. Although MSA-P patients are younger with theoretical age-related benefits in gait performance we observed a profound alteration of average and kinematic gait metrics that are clearly linked to the severe motor and gait impairment of the disease.

Intriguingly, while the average stride length displayed by PD patients was significantly reduced compared to controls, the same did not apply for the variability kinematics, where no significant difference was observed. These measures are apparently in contrast with previously published data, where PD show higher GV than healthy controls [8, 25]. Hausdorff et al. described an increased variability in PD patients [8] but the control group was significantly younger than the PD cohort and $60 \%$ of PD patients were in a severe disease stage (Hoehn and Yahr $\geq 3$ ). Similar results were described by Rennie et al. [25], where the control group was not age matched. Our results indicate that even when gait speed changes, variability in PD and controls at the same age show similar gait stability. It is largely known that mechanical and energy expenditure optimizations change over the lifespan [26] and the effects of aging may also play a role here. That said, the impairment of average stride length displayed by PD patients may be influenced by an altered locomotor control system affecting gait stability and regularity $[8,27]$ or rather be a consequence of bradykinesia and lower gait speed, not intrinsic to the disease [28]. Furthermore, the fact that all our PD patients were evaluated in a stable ON phase may have positively affected gait rhythmicity, putting forth the hypothesis that dopaminergic therapy plays an important role in maintaining gait stability in PD [10].

The within-group analysis between the three walking velocities revealed an increased gait variability by reducing the gait velocity in MSA-P patients, while PD and controls showed the lowest variability at a comfortable speed. Here, it must be mentioned that all groups were able to adapt walking velocity without external cueing, as presented in significant within-group differences in gait velocity between the three conditions. Previous observations described minimal variability of gait at self-paced comfortable speed in healthy adults [29]. Indeed, this might be the walking condition with the most efficient gait and minimal metabolic energy costs. However, MSA-P patients show numerically the lowest variability in fast speed and a significant increase in CV parameters between comfortable and slow velocity. This finding supports the hypothesis that walking slowly is a more challenging task unveiling postural instability in MSA-P patients and speed modulation might not entirely be controlled by a single mechanism [8]. Potential changes in specific basal ganglia loops for integrating sensory stimuli, regulating muscle tone and performing automatic sequential movements may play a role here, but need to be further investigated.

Finally, the correlation analysis of the gait parameters with MDS-UPDRS III revealed positive moderate to strong correlations for all CV parameters in MSA-P-mainly in comfortable and slow speed, while PD patients showed hardly any correlation. Consistent with recent findings from Hasegawa et al. [30], these result may be due to the fact that the MDS-UPDRS motor score includes-beside axial-symptoms such as hypo/bradykinesia, rigidity or limb tremor, which are not closely linked with the gait metrics. Corroborating this hypothesis, a correlation analysis with the gait-focused PIGD subitems revealed significant correlations in both disease groups. 


\section{Limitations}

There are some limitations to our study. The patients' cohorts were rather small due to the fact that MSA-P is an orphan disease and recruitment of patients who are able to walk independently without aids is challenging. Therefore, results should be interpreted with caution. However, the patients' cohorts were well characterized and similar with regard to global motor disability. In addition, post-mortem neuropathological evaluation was not available and misdiagnosis cannot be ruled out. To minimize this problem, the clinical examination was performed by a movement disorder specialist according to the existing diagnostic criteria and patients were followed up for at least 2 years to reduce likelihood of misdiagnoses. One patient was revised from MSA-P to PD after two years, the diagnosis of all other subjects remained the same until this post-hoc analysis. MSA-P patients were significantly younger compared to the other groups. However, gait impairment in MSA-P outweighs age effects what we confirmed in a covariate analysis.

\section{Conclusion}

To the best of our knowledge, this study was the first of its kind to evaluate gait kinematics in patients with MSA-P at different walking speed conditions. Comparing those values with PD and elderly controls free of parkinsonism and gait disorders measured by the same wearable system and with the same standardized set-ups should be acknowledged as a strength of this study, too. The present study aims to be an initial step towards gaining a more all-embracing understanding of the mechanisms that underlie gait variability in MSA-P and parkinsonism in general. Our data suggest that sensor-derived gait parameters discriminate MSA-P from $\mathrm{PD}$ and correlate with clinical rating scales and, therefore, provide objective research outcomes. Inertial sensors have the potential to continuously monitor gait impairment under real-life conditions of patients instead of generating snapshots in short-lasting doctoral visits. Our long-term goal is to support the visits in the hospital by continuous data of real-life scenarios to draw a holistic picture of gait impairment in everyday life. However, more research is required to determine the trade-off between validity and clinical utility of the gait variability kinematics, its dynamics and responses to interventions.

Acknowledgements We thank our patients and families and the MSA Coalition for supporting this research. This work was supported by the Bavarian Ministry for Economy, Regional Development and Energy via the Medical Valley Award 2017 (FallRiskPD Project) and EIT Health. $\mathrm{BE}, \mathrm{JK}$, and HG are supported by Mobilise-D from the Innovative Medicines Initiative 2 Joint Undertaking under grant agreement no. 820820. Furthermore, we are grateful for the support of VASCage
(Research centre on vascular ageing and stroke, Innsbruck, Austria) for supporting the recruitment of our control group.

Author contributions All authors contributed to the study conception and design. Material preparation, data collection and analysis were performed by VS, CR, CK, SE, AF, RG, KS, PM, HS, KM, OS, JK, GW, and HG. The first draft of the manuscript was written by VS, CR and HG. All authors commented on previous versions of the manuscript. All authors read and approved the final manuscript.

Funding Open access funding provided by University of Innsbruck and Medical University of Innsbruck. This was an academic and not an industry supported study. This work was performed at the Department of Neurology, Innsbruck Medical University, Innsbruck, Austria and the Bruneck hospital in Bruneck, Italy. Assessment of the control group was supported by a research grant from the Tiroler Wirtschaftsförderung [Grant Number UNI-0404/2245].

\section{Compliance with ethical standards}

Conflicts of interest Victoria Sidoroff: none. Cecilia Raccagni: none. Christine Kaindlstorfer: none. Sabine Eschlboeck: none. Alessandra Fanciulli: none concerning this project. A.F. reports royalties from springer Nature, speaker fees and honoraria from the Ordensklinikum Linz, Austrian Parkinson Society, International Parkinson Disease and Movement Disorder Society and Theravance Biopharma and research grant from Stitching Parkinson Fond and the Österreichischer Austauschdienst outside of the submitted work. Roberta Granata: none. Björn Eskofier: none concerning this project. B.E. is supported by Mobilise-D from the Innovative Medicines Initiative 2 Joint Undertaking under grant agreement no. 820820. He further received an institutional research grant from the Bavarian Ministry of Economic Affairs and Media, Energy and Technology, Germany (Medical Valley Award, FallRiskPD). Klaus Seppi: none concerning this project. K.S. reports personal fees from Teva, UCB, Lundbeck, AOP Orphan Pharmaceuticals AG, Roche, Grünenthal and Abbvie, honoraria from the International Parkinson and Movement Disorders Society, research grants from FWF Austrian Science Fund, Michael J. Fox Foundation, and International Parkinson and Movement Disorder Society, outside the submitted work. Werner Poewe: none concerning this project. W.P. reports personal fees from AbbVie, Affiris, AstraZeneca, BIAL, Britannia, Intec, Ipsen, Lundbeck, Neuroderm, Denali Pharmaceuticals, Novartis, Orion Pharma, Roche, Teva, UCB and Zambon (consultancy and lecture fees in relation to clinical drug development programmes for $P D$ ). Johann Willeit: none. Stefan Kiechl: none concerning this project. S.K. is CSO of VASCage, a COMET Research Centre of the Austria Research Promotion Agency. Philipp Mahlknecht: none. Heike Stockner: none. Kathrin Marini: K. M. was supported by a research grant from the Tiroler Wirtschaftsförderung (grant number UNI-0404/2245). Oliver Schorr: none. Gregorio Rungger: none. Jochen Klucken: none concerning this project. J.K. is supported by Mobilise-D from the Innovative Medicines Initiative 2 Joint Undertaking under grant agreement no. 820820. He further received an institutional research grant from the Bavarian Ministry of Economic Affairs and Media, Energy and Technology, Germany (Medical Valley Award, FallRiskPD) and reports institutional research grants from EITHealth; EIT-Digital; EU (H2020), German Research Foundation (DFG). Industry sponsored institutional IITs and grants from Alpha-Telemed AG. Compensation and honoraria from serving on scientific advisory boards and lecturing from Ever Neuro Pharma GmbH, TEVA Pharma $\mathrm{GmbH}$, Bial Deutschland $\mathrm{GmbH}$; Celgene $\mathrm{GmbH}$, and Magisan $\mathrm{GmbH}$. Gregor K. Wenning: none concerning this project. G.W. reports personal fees from Biohaven, Theravance, UCB, Lundbeck, and Ono, honoraria from the Austrian Autonomic Society, research grants from FWF Austrian Science Fund, International Parkinson and Movement Disorder 
Society, and US MSA Coalition, outside of the submitted work. Heiko Gaßner: none concerning this project. H.G. is supported by Mobilise-D from the Innovative Medicines Initiative 2 Joint Undertaking under grant Agreement No. 820820. He further received an institutional research grant from the Bavarian Ministry of Economic Affairs and Media, Energy and Technology, Germany (Medical Valley Award, FallRiskPD). HG received an institutional research grant by the Federal Ministry of Education and Research (project: treatHSP) and Huntington-Stiftung of the Deutsche Huntington Hilfe e.V. HG received further support by the Medical Research Foundation at the University Hospital Erlangen and the Förderverein für HSP-Forschung e.V. outside of the submitted work.

Ethical standards All procedures performed in studies involving human participants were in accordance with the ethical standards of the institutional and/or national research committee and with the 1975 Helsinki Declaration and its later amendments or comparable ethical standards. Informed consent was obtained from all the patients included in the study. The study was approved by local ethics committees (IRB-approval-No 0365, 344/4.25 378/5.3, 20.10.2017).

Consent to participate All study participants gave written informed consent for participation and publication prior to participation according to the Declaration of Helsinki.

Open Access This article is licensed under a Creative Commons Attribution 4.0 International License, which permits use, sharing, adaptation, distribution and reproduction in any medium or format, as long as you give appropriate credit to the original author(s) and the source, provide a link to the Creative Commons licence, and indicate if changes were made. The images or other third party material in this article are included in the article's Creative Commons licence, unless indicated otherwise in a credit line to the material. If material is not included in the article's Creative Commons licence and your intended use is not permitted by statutory regulation or exceeds the permitted use, you will need to obtain permission directly from the copyright holder. To view a copy of this licence, visit http://creativecommons.org/licenses/by/4.0/.

\section{References}

1. Gassner H, Raccagni C, Eskofier BM, Klucken J, Wenning GK (2019) The diagnostic scope of sensor-based gait analysis in atypical Parkinsonism: further observations. Front Neurol 10:5. https ://doi.org/10.3389/fneur.2019.00005

2. Fanciulli A, Goebel G, Lazzeri G, Scherfler C, Gizewski ER, Granata R et al (2019) Early distinction of Parkinson-variant multiple system atrophy from Parkinson's disease. Mov Disord 34(3):440-441. https://doi.org/10.1002/mds.27635

3. Hamacher D, Singh NB, Van Dieën JH, Heller MO, Taylor WR (2011) Kinematic measures for assessing gait stability in elderly individuals: a systematic review. J R Soc Interface 8(65):16821698. https://doi.org/10.1098/rsif.2011.0416

4. Raccagni C, Goebel G, Gaßner H, Granata R, Ndayisaba JP, Seebacher B et al (2019) Physiotherapy improves motor function in patients with the Parkinson variant of multiple system atrophy: a prospective trial. Parkinsonism Relat Disord 67:60-65. https:// doi.org/10.1016/j.parkreldis.2019.09.026

5. Klucken J, Barth J, Kugler P, Schlachetzki J, Henze T, Marxreiter F et al (2013) Unbiased and mobile gait analysis detects motor impairment in Parkinson's disease. PLoS ONE 8(2):e56956. https ://doi.org/10.1371/journal.pone.0056956

6. Schlachetzki JCM, Barth J, Marxreiter F, Gossler J, Kohl Z, Reinfelder S et al (2017) Wearable sensors objectively measure gait parameters in Parkinson's disease. PLoS ONE 12(10):e0183989. https://doi.org/10.1371/journal.pone.0183989

7. Hausdorff JM (2005) Gait variability: methods, modeling and meaning. J Neuroeng Rehabil 2:19. https://doi. org/10.1186/1743-0003-2-19

8. Hausdorff JM, Cudkowicz ME, Firtion R, Wei JY, Goldberger AL (1998) Gait variability and basal ganglia disorders: stride-to-stride variations of gait cycle timing in Parkinson's disease and Huntington's disease. Mov Disord 13(3):428-437. https://doi.org/10.1002/ mds. 870130310

9. Brach JS, Perera S, Studenski S, Katz M, Hall C, Verghese J (2010) Meaningful change in measures of gait variability in older adults. Gait Posture 31(2):175-179. https://doi.org/10.1016/j.gaitp ost.2009.10.002

10. Schaafsma JD, Giladi N, Balash Y, Bartels AL, Gurevich T, Hausdorff JM (2003) Gait dynamics in Parkinson's disease: relationship to Parkinsonian features, falls and response to levodopa. J Neurol Sci 212(1-2):47-53

11. Raccagni C, Gaßner H, Eschlboeck S, Boesch S, Krismer F, Seppi $\mathrm{K}$ et al (2018) Sensor-based gait analysis in atypical parkinsonian disorders. Brain Behav 8(6):e00977. https://doi.org/10.1002/ brb3.977

12. Na BS, Ha J, Park JH, Ahn JH, Kim M, Kim JS et al (2019) Comparison of gait parameters between drug-naïve patients diagnosed with multiple system atrophy with predominant parkinsonism and Parkinson's disease. Parkinsonism Relat Disord 60:87-91. https ://doi.org/10.1016/j.parkreldis.2018.09.018

13. Borm C, Krismer F, Wenning GK, Seppi K, Poewe W, Pellecchia MT et al (2018) Axial motor clues to identify atypical parkinsonism: a multicentre European cohort study. Parkinsonism Relat Disord 56:33-40. https://doi.org/10.1016/j.parkreldis.2018.06.015

14. Aerts MB, Abdo WF, Bloem BR (2011) The "bicycle sign" for atypical parkinsonism. Lancet 377(9760):125-126. https://doi. org/10.1016/S0140-6736(11)60018-4

15. Postuma RB, Berg D, Stern M, Poewe W, Olanow CW, Oertel W et al (2015) MDS clinical diagnostic criteria for Parkinson's disease. Mov Disord 30(12):1591-1601. https://doi.org/10.1002/ mds. 26424

16. Gilman S, Wenning GK, Low PA, Brooks DJ, Mathias CJ, Trojanowski JQ et al (2008) Second consensus statement on the diagnosis of multiple system atrophy. Neurology 71(9):670-676. https ://doi.org/10.1212/01.wnl.0000324625.00404.15

17. Goetz CG, Tilley BC, Shaftman SR, Stebbins GT, Fahn S, Martinez-Martin P et al (2008) Movement Disorder Society-sponsored revision of the Unified Parkinson's Disease Rating Scale (MDSUPDRS): scale presentation and clinimetric testing results. Mov Disord 23(15):2129-2170. https://doi.org/10.1002/mds.22340

18. Wenning GK, Tison F, Seppi K, Sampaio C, Diem A, Yekhlef F et al (2004) Development and validation of the Unified Multiple System Atrophy Rating Scale (UMSARS). Mov Disord 19(12):1391-1402. https://doi.org/10.1002/mds.20255

19. Curtze C, Nutt JG, Carlson-Kuhta P, Mancini M, Horak FB (2015) Levodopa is a double-edged sword for balance and gait in people with Parkinson's disease. Mov Disord 30(10):1361-1370. https:// doi.org/10.1002/mds.26269

20. Kiechl S, Willeit J (2019) In a Nutshell: findings from the Bruneck study. Gerontology 65(1):9-19. https://doi.org/10.1159/00049 2329

21. Mahlknecht P, Gasperi A, Djamshidian A, Kiechl S, Stockner H, Willeit P et al (2018) Performance of the Movement Disorders Society criteria for prodromal Parkinson's disease: a populationbased 10-year study. Mov Disord 33(3):405-413. https://doi. org/10.1002/mds. 27281

22. Barth J, Oberndorfer C, Pasluosta C, Schülein S, Gassner H, Reinfelder S et al (2015) Stride segmentation during free walk movements using multi-dimensional subsequence dynamic time 
warping on inertial sensor data. Sensors (Basel) 15(3):6419-6440. https://doi.org/10.3390/s150306419

23. Caderby T, Yiou E, Peyrot N, Begon M, Dalleau G (2014) Influence of gait speed on the control of mediolateral dynamic stability during gait initiation. J Biomech 47(2):417-423. https://doi. org/10.1016/j.jbiomech.2013.11.011

24. Bryant MS, Rintala DH, Hou JG, Charness AL, Fernandez AL, Collins RL et al (2011) Gait variability in Parkinson's disease: influence of walking speed and dopaminergic treatment. Neurol Res 33(9):959-964. https://doi.org/10.1179/1743132811Y.00000 00044

25. Rennie L, Dietrichs E, Moe-Nilssen R, Opheim A, Franzen E (2017) The validity of the Gait Variability Index for individuals with mild to moderate Parkinson's disease. Gait Posture 54:311317. https://doi.org/10.1016/j.gaitpost.2017.03.023

26. Malatesta D, Simar D, Dauvilliers Y, Candau R, Borrani F, Prefaut $C$ et al (2003) Energy cost of walking and gait instability in healthy 65- and 80-yr-olds. J Appl Physiol (1985) 95(6):22482256. https://doi.org/10.1152/japplphysiol.01106.2002
27. Blin O, Ferrandez AM, Serratrice G (1990) Quantitative analysis of gait in Parkinson patients: increased variability of stride length. J Neurol Sci 98(1):91-97. https://doi.org/10.1016/0022510x(90)90184-o

28. Frenkel-Toledo S, Giladi N, Peretz C, Herman T, Gruendlinger L, Hausdorff JM (2005) Effect of gait speed on gait rhythmicity in Parkinson's disease: variability of stride time and swing time respond differently. J Neuroeng Rehabil 2:23. https://doi. org/10.1186/1743-0003-2-23

29. Sekiya N, Nagasaki H, Ito H, Furuna T (1997) Optimal walking in terms of variability in step length. J Orthop Sports Phys Ther 26(5):266-272. https://doi.org/10.2519/jospt.1997.26.5.266

30. Hasegawa N, Shah VV, Carlson-Kuhta P, Nutt JG, Horak FB, Mancini M (2019) How to select balance measures sensitive to Parkinson's disease from body-worn inertial sensors-separating the trees from the forest. Sensors (Basel). https://doi.org/10.3390/ s19153320

\section{Affiliations}

\section{Victoria Sidoroff $^{1} \cdot$ Cecilia Raccagni $^{1,6}\left(\mathbb{D} \cdot\right.$ Christine Kaindlstorfer $^{1} \cdot$ Sabine Eschlboeck $^{1} \cdot$ Alessandra Fanciulli $^{1}$. Roberta Granata $^{1} \cdot$ Björn Eskofier ${ }^{2} \cdot$ Klaus Seppi $^{1}$ - Werner Poewe ${ }^{1} \cdot$ Johann Willeit ${ }^{1}$. Stefan Kiechl ${ }^{1}$. Philipp Mahlknecht ${ }^{1} \cdot$ Heike Stockner $^{1} \cdot$ Kathrin Marini $^{1}$. Oliver Schorr ${ }^{1}$ - Gregorio Rungger ${ }^{3}$. Jochen Klucken ${ }^{4,5}$. Gregor Wenning ${ }^{1} \cdot$ Heiko Gaßner ${ }^{4}$}

Heiko Gaßner

heiko.gassner@uk-erlangen.de

1 Department of Neurology, Medical University of Innsbruck, Innsbruck, Austria

2 Machine Learning and Data Analytics Lab, Friedrich-Alexander University Erlangen-Nürnberg (FAU), Erlangen, Germany

3 Department of Neurology, Hospital of Bruneck, Bruneck, Italy
4 Department of Molecular Neurology, Universitätsklinikum Erlangen, Friedrich-Alexander University, Erlangen-Nürnberg (FAU), Schwabachanlage 6, 91054 Erlangen, Germany

5 AG Digital Health Pathways, Fraunhofer Institute for Integrated Circuits, Erlangen, Germany

6 Department of Neurology, Regional General Hospital Bolzano, Lorenz Boehler Street 5, 39100 Bolzano, Italia 